\title{
PRODUÇÃO CIENTÍFICA EM GESTÃO DE CUSTOS EM HOSPITAIS: UMA ANÁLISE NOS PRINCIPAIS EVENTOS ACADÊMICOS NA ÁREA CONTÁBIL NO PERÍODO DE 2007 A 2011 ${ }^{1}$
}

\author{
SCIENTIFIC PRODUCTION ON COST MANAGEMENT IN HOSPITALS: AN \\ ANALYSIS OF THE MAIN ACADEMIC ACCOUNTING EVENTS BETWEEN 2007 \\ AND 2011
}

\section{GESTIÓN DE COSTOS DE PRODUCCIÓN CIENTÍFICA EN LOS HOSPITALES: UN ANÁLISIS EN PRINCIPALES EVENTOS ACADÉMICOS EN CONTABILIDAD PERÍODO DE 2007 A 2011}

Maria Eliana dos Santos

m.elianasantos@yahoo.com.br

Davy Antonio da Silva

davyantonio@yahoo.com.br
Edvalda Araújo Leal

edavalda@facic.ufu.br

\section{Resumo:}

O objetivo deste artigo é identificar o perfil das pesquisas desenvolvidas e publicadas sobre o tema gestão de custos, com ênfase na área hospitalar. Para tanto, realizou-se um levantamento bibliométrico das pesquisas publicadas nos principais eventos na área de contabilidade no Brasil (EnANPAD; AnpCont; USP e Congresso de Custos), no período de 2007 a 2011. A metodologia de pesquisa adotada constitui-se de um estudo bibliométrico, realizado por meio da pesquisa descritiva, com abordagem qualitativa. Os resultados identificaram 40 artigos científicos publicados na área de custos com ênfase no setor hospitalar. Dos eventos pesquisados, o Congresso Brasileiro de Custos foi o que apresentou o maior número de publicações. Na classificação das publicações por origem institucional dos autores, verificou-se que o maior volume refere-se às publicações de autores vinculados à Universidade Federal de Minas Gerais, com destaque também para a Universidade Federal do Rio Grande do Norte. Verificou-se, também, que $95 \%$ dos artigos investigados utilizaram a aplicação empírica, o que reforça o interesse dos autores em verificar a aplicabilidade da gestão de custos nas organizações hospitalares.

Palavras-chave: Estudo bibliométrico; Gestão de custos; Hospital;

\begin{abstract}
:
This article aims to identify and profile the research done and published on hospital cost management. Therefore, a bibliometric survey was carried out on the research published for the main academic accounting events in Brazil (EnANPAD; AnpCont; USP and the Brazilian Cost Conference) from 2007 to 2011. The bibliometric study was done through qualitative descriptive research. Results have identified 40 papers published on hospital cost management. Of the events studied, the Brazilian Cost Conference had the highest number of publications on the subject. As for classifying the publications by institutional origin of the authors, the highest volume was from the Federal University of Minas Gerais, followed by the Federal University of Rio Grande do Norte. It was found that $95 \%$ of the studied articles used empirical application, which reinforces the authors' interest in verifying the applicability of hospital cost management.
\end{abstract}

Keywords: Bibliometric study; Cost management; Hospitals;

\section{Resumen}

El propósito de este artículo es identificar el perfil de la investigación desarrollada y publicada sobre el tema de la gestión de costes, con énfasis en el área hospitalaria. Para ello, se realizó una encuesta bibliométrico de la

\footnotetext{
${ }^{1}$ Submetido em 16 de outubro de 2012. Aceito em 04 de dezembro de 2013. O artigo foi avaliado segundo o processo de duplo anonimato além de ser avaliado pelo editor. Editores responsáveis: Márcio Augusto Gonçalves e Lucas Maia dos Santos. Reprodução parcial ou total e trabalhos derivativos permitidos com a citação apropriada da fonte.
} 
investigación publicada en los principales acontecimientos en el campo de la contabilidad en el Brasil ( EnANPAD ; AnpCont ; USP y el Congreso costos ) durante el período 2007-2011. La metodología de investigación utilizada consistió en un estudio bibliométrico , por medio de la investigación descriptiva con enfoque cualitativo. Los resultados identificaron 40 artículos científicos publicados en el área de costos con énfasis en el sector hospitalario. Eventos encuestados, el Congreso Brasileño de costos se presentó el mayor número de publicaciones. En la clasificación de publicaciones de origen institucional de los autores, se encontró que el mayor volumen se refiere a las publicaciones de autores vinculados a la Universidad Federal de Minas Gerais , en especial también para la Universidad Federal de Rio Grande do Norte . También se constató que el $95 \%$ de los artículos estudiados utiliza la aplicación empírica, lo que refuerza el interés de los autores para verificar la aplicabilidad de la gestión de costos en las organizaciones hospitalarias .

Palabras clave : Estudio bibliométrico, costos de gestión; hospitalarios;

\section{INTRODUÇÃO}

Com o avanço da globalização no mercado brasileiro e a alta competitividade enfrentada pelas organizações, é fundamental, para os hospitais, uma administração eficaz e eficiente. A busca pelo menor custo sem afetar a qualidade e a funcionalidade dos serviços é um objetivo permanente das empresas que buscam obter vantagens no mercado.

As instituições hospitalares são organizações complexas que atuam na área de serviços de internação, prevenção, pesquisa, tratamento e diagnóstico. Segundo Campos e Marques (2011, p.2), "para que um hospital possa atingir excelência na prestação de seus serviços, é essencial que realize constantes investimentos, melhore sua estrutura física, adéqüe tecnologicamente os materiais e equipamentos". Entretanto, grande parte das organizações hospitalares, em nosso país, não faz uso de uma gestão adequada de custos que seja compatível com as necessidades hospitalares, de modo a orientar e oferecer parâmetros para suas decisões administrativas e o controle de atividades (ABBAS, 2001).

A gestão de custos nas organizações hospitalares permite comparar desempenho e objetivos, além de analisar possibilidades de redução de custos e evidenciar ineficiências de serviços e processos. A complexidade das instituições hospitalares padece com a busca de um sistema de custos ideal para as atividades desenvolvidas, especificas de sua atuação. Falk (2001) observa que o controle de custos hospitalares tem recebido pouco espaço na literatura.

Lucena, Freire e Brito (2008), ao buscar identificar o perfil das publicações na área de custos voltados para a área hospitalar, publicados nos anais do congresso USP, realizado nos períodos de 2001 a 2007, evidenciaram a carência de estudos em custos voltados para a área hospitalar.

Nesse contexto, para dar continuidade à pesquisa feita por Lucena, Freire e Brito (2008), o presente estudo tem como objetivo identificar o perfil das pesquisas desenvolvidas e publicadas sobre o tema gestão de custos, com ênfase na área hospitalar. Realizou-se um levantamento bibliométrico das pesquisas publicadas nos principais eventos na área de contabilidade no Brasil, no período de 2007 a 2011. A pesquisa permitiu, também, evidenciar quais foram as abordagens metodológicas adotadas nos trabalhos publicados.

A estrutura deste trabalho é composta por cinco seções, contando com esta introdução. A seção dois apresenta o referencial teórico para a fundamentação do estudo. Na sequência, apresentam-se os procedimentos metodológicos adotados no estudo. Na quarta seção, evidenciam-se os principais resultados da pesquisa, e, por último, as considerações finais referentes aos resultados alcançados.

\section{REFERENCIAL TEÓRICO}




\subsection{A RELEVÂNCIA DA PUBLICAÇÃO CIENTÍFICA}

Pode-se definir pesquisa como o processo formal e sistemático de desenvolvimento do método científico. $\mathrm{O}$ objetivo fundamental da pesquisa é descobrir respostas para problemas, mediante o emprego de procedimentos científicos (GIL, 2011). A pesquisa busca gerar e ampliar conhecimento científico sobre determinado assunto por meio da comunicação.

Nesse contexto, a finalidade da pesquisa científica não consiste somente na produção de um relatório ou descrição de fatos levantados empiricamente, mas, sim, no desenvolvimento de um caráter interpretativo no que se refere aos dados obtidos. Para tal, é imprescindível correlacionar a pesquisa com o universo teórico, optando-se por um modelo teórico que sirva de embasamento à interpretação do significado dos dados e fatos colhidos ou levantados (LAKATOS e MARCONI, 1991, p. 224 apud MACHADO e SILVA, 2007, p. 4).

Uma das possibilidades de avaliar a produção científica é a utilização de métodos que permitam medir a produtividade dos pesquisadores, grupos ou instituições de pesquisas. Para tanto, torna-se fundamental o uso de técnicas quantitativas e qualitativas, ou mesmo uma combinação entre ambas. Com a necessidade de quantificar fenômenos, surge a bibliometria que, até a década de1960, era conhecida como bibliografia estatística (termo cunhado por Edward Wyndham Hulme em 1922), sendo popularizada por Alan Pritchard, em 1969, pelo uso do termo Bibliometria. Definiu-se, então, bibliometria como a aplicação de métodos matemáticos e estatísticos dos livros e outros meios de comunicação, sendo sua utilização sugerida em todos os estudos que se proponham a quantificar o processo de comunicação escrita. De forma literal, Bibliometria significa "medida do livro". O que se pretende medir com a bibliometria são os padrões estatísticos encontrados em diversos tipos de publicação, não se limitando aos livros. Esses padrões estatísticos referem-se a variáveis, como autoria, fonte, assunto, origem geográfica e citações (BIRGER HJORLAND, 2005).

Atualmente, o campo da bibliometria, de modo geral, inclui todos os aspectos quantitativos, bem como os modelos da comunicação científica e do armazenamento, disseminação e recuperação da informação científica. Esse conceito de bibliometria é muito mais amplo do que as definições usuais do termo, objetivando incorporar todas as orientações correntes, como suas aplicações à política científica, à biblioteconomia e à recuperação da informação (WORMELL, 1998, p. 211).

No próximo tópico, apresentam-se estudos na área de custos nos quais foram utilizados a bibliometria.

\subsection{ESTUDOS CORRELATOS: PERFIL DAS PESQUISAS NA ÁREA DE CUSTOS}

Nesta pesquisa, foram identificados estudos que apresentaram o perfil dos trabalhos científicos na área de custos. Cardoso, Pereira e Guerreiro (2004), por exemplo, traçaram um perfil da pesquisa em custos no âmbito da temática de contabilidade e controle gerencial do EnANPAD, desde a sua inauguração como área autônoma, em 1998, até 2003. Os resultados evidenciaram um forte crescimento quantitativo da temática de contabilidade, elevando do patamar de 40 trabalhos submetidos em 1998 para 191 trabalhos, em 2003. Esse crescimento quantitativo não foi acompanhado, na mesma proporção, pelo crescimento qualitativo dos trabalhos na área de custos. 
O estudo feito por Callado e Almeida (2005) apresentou o perfil da produção acadêmica, no âmbito do Congresso Brasileiro de Custos, no período de 1994 a 2004. Os resultados encontrados pelos autores indicam que as investigações acadêmicas estão voltadas para os modelos de mensuração, tendo sido realizadas, predominantemente, nas regiões Sudeste e Sul, abrangendo, em sua maioria, aspectos relacionados à exploração zootécnica como atividade econômica estudada. Outro aspecto relevante identificado é a elevada participação relativa dos artigos elaborados a partir de revisões da literatura e pesquisas bibliográficas.

Schultz, Borget e Laffin (2006) classificaram a produção científica na área de custos no setor de serviços, por meio da análise das publicações de pesquisas no EnANPAD e em periódicos na área de administração, durante o período de 2000 a 2004, chegando a resultados que evidenciam a escassez de trabalhos nesta área. Nesse estudo, não foram identificados autores, nem grupos de pesquisa ou mesmo entidades focadas em pesquisas neste assunto, embora o número total de artigos publicados, tanto no EnANPAD quanto em periódicos, tenha apresentado um crescimento no volume de publicações.

Já com o objetivo de verificar o perfil das publicações na área de custos voltados para a área hospitalar, Lucena, Freire, Silva e Brito (2008) investigaram os anais do congresso USP de controladoria e contabilidade, realizados nos períodos de 2001 a 2007. Os resultados do estudo indicaram que os temas aplicados a custos hospitalares se concentraram na temática controladoria e contabilidade gerencial, sendo a USP a instituição que mais publicou trabalhos de custos na área hospitalar. Os livros são as principais fontes de pesquisa utilizadas, apresentando um percentual de 56,30\%. Devido à pequena quantidade de artigos publicados na área de custos hospitalares, não foi possível identificar uma instituição, grupo de pesquisa ou autor que se destaque quanto ao número de publicações voltadas para o tema objeto de estudo do artigo. Por fim, os autores observaram que, em relação ao número de artigos de custos publicados, percebeu-se uma forte tendência de, até o ano de 2010, não haver nenhuma publicação na área de custos hospitalares, no congresso da USP.

A pesquisa realizada por Rocha, Reis, Souza, Cruz e Tracz (2010) teve como objetivo analisar as publicações voltadas para a abordagem de gestão de custos no EnANPAD. Como resultado, pode-se observar que, dos pesquisadores analisados, apenas 9 tiveram três ou mais artigos publicados, respondendo por mais de $27,34 \%$ do total de artigos. Com relação ao mapeamento dos elos relacionais entre os autores, verificou-se uma baixa densidade geral no período de 1997-2002 (0,0183) e no período de 2003-2008 (0,0115), cercada por baixa média de centralidade, evidenciando uma suposta relação embrionária no desenvolvimento de cooperações entre pesquisadores das temáticas de Gestão de Custos no EnANPAD, nos últimos 12 anos.

Júnior, Araújo, e Rezende (2010) realizaram um estudo bibliométrico, no período de 2007 a 2009, da área ensino e pesquisa na gestão de custos, a qual integra o Congresso Brasileiro de Custos. Os resultados identificaram 49 artigos na área "ensino e pesquisa em gestão de custo". Ainda, foram identificadas 36 instituições de ensino as quais os autores tiveram seus trabalhos vinculados.

Quanto a trabalhos relacionados diretamente com a análise de publicações na área de custos em hospitais, foi encontrado somente o trabalho de Lucena, Freire, Silva e Brito (2008). Dessa forma, o presente estudo propõe-se a ampliar a amostra e o período investigado da pesquisa desses autores. 


\title{
2.3 CONTABILIDADE DE CUSTOS NAS ENTIDADES HOSPITALARES
}

A organização hospitalar é uma das mais complexas, não apenas pela nobreza e amplitude da sua missão, mas, sobretudo, por apresentar uma equipe multidisciplinar com elevado grau de autonomia para dar assistência à saúde em caráter preventivo, curativo e reabilitador aos pacientes, em regime de internação, no qual se utiliza tecnologia de ponta e de rotina, de forma crescente (AZEVEDO, 1993).

Almeida (1983, p.205) conceitua hospital como:

\begin{abstract}
"Uma instituição destinada ao diagnóstico e tratamento de doentes internos e externos; planejada e construída ou modernizada com orientação técnica; bem organizada e convenientemente administrada consoante padrões e normas estabelecidas, oficial ou particular, com finalidades diversas [...] servindo ao mesmo tempo para prevenir contra a doença e promover a saúde, a prática, a pesquisa e o ensino da medicina e da cirurgia, da enfermagem e da dietética, e das demais especialidades afins".
\end{abstract}

Para a prestação de serviços hospitalares, são necessários investimentos contínuos em ativos fixos e tecnológicos, bem como o emprego intensivo de profissionais das mais diversas áreas do conhecimento (SILVA; BORGET e SCHULTZ, 2009).

As informações mais detalhadas de custos em hospitais oferecem importantes esclarecimentos sobre o comportamento dos custos em relação a variações no volume de serviços, permitindo melhor gerenciamento de custos em nível departamental, além de possibilitar a identificação e elaboração de estratégias eficazes para o controle mais preciso dos custos, permitindo melhor análise da lucratividade dos diversos serviços prestados (FALK, 2001; CHING, 2010).

Desconhecer os custos pode agravar a posição financeira presente e futura do hospital. A descrição e o conhecimento dos custos são importantes, pois sua atribuição de forma correta pode auxiliar no planejamento financeiro dos hospitais e, também, de outras empresas, sejam elas de serviços ou industriais (MARTINS, 2000).

Segundo Abbas (2001), um percentual significativo de gestores hospitalares utiliza sistemas de custos tradicionais, não obtendo todas as informações necessárias para auxiliá-los no processo de tomada de decisões. Matos (2002, p. 197) considera que "a metodologia de apropriação de custos dos procedimentos hospitalares propicia inegáveis benefícios ao processo de gestão". Nesse sentido, o autor destaca que a gestão de custos permite o agrupamento dos procedimentos hospitalares por especialidades médicas, facilitando a análise de resultados do hospital, além de facilitar o controle dos custos e recursos utilizados e possibilitar comparações com outras instituições, quanto à qualidade, custos e preço dos tratamentos, consistindo em uma referência para o estabelecimento de preços sob a metodologia dos pacotes de prestação de serviços médico-hospitalares.

Verifica-se, nesse contexto, a relevância da pesquisa na área hospitalar, principalmente, no levantamento dos estudos já realizados nas instituições relacionadas ao tema gestão de custos.

Na sequência, apresentam-se os procedimentos metodológicos aplicados na realização da pesquisa. 


\section{PROCEDIMENTOS METODOLÓGICOS}

\subsection{CARACTERIZAÇÃO DO MÉTODO}

Este estudo classifica-se como uma pesquisa descritiva, em razão do objetivo que visa identificar, analisar e classificar as características de um grupo de publicações em gestão de custos, com ênfase na área Hospitalar. Segundo Gil (2011, p.28), "este tipo de pesquisa tem como principal objetivo descrever as características de determinada população ou fenômeno ou ainda estabelecer relações entre as variáveis".

Para o levantamento das informações, utilizaram-se dados secundários coletados em cinco edições de cinco eventos nacionais, quais sejam: Encontro da Associação Nacional de Pós-Graduação e Pesquisa em Administração (EnANPAD), Congresso USP de Controladoria e Contabilidade, Congresso USP de Iniciação Científica em Contabilidade, Congresso Brasileiro de Custos (CBC) e Congresso da Associação Nacional dos Programas de PósGraduação em Ciências Contábeis (ANPCONT). Esses eventos foram escolhidos por atenderem ao critério estabelecido, nesta pesquisa, que é possuir classificação E1 ou E2, de acordo com o sistema Qualis da CAPES.

Quanto à abordagem do problema, esta pesquisa classifica-se como qualitativa, visto que "os estudos que empregam uma metodologia qualitativa podem descrever a complexidade de determinado problema, analisar a interação de certas variáveis, compreender e classificar processos dinâmicos vividos por grupos sociais" (RICHARDSON, 1999 p.80).

O estudo caracteriza-se como bibliométrico, pois teve o objetivo de quantificar a comunicação científica escrita, utilizando processos variados de levantamento, tratamento e apresentação de dados.

\subsection{Coleta dos Dados e Amostra Selecionada}

O universo desta pesquisa é constituído por todos os artigos publicados nos anais dos congressos classificados como E1 e E2 no sistema Qualis da CAPES, quais sejam: CBC, EnANPAD, Congresso USP e ANPCONT, no período de 2007 a 2011.

Os artigos analisados foram obtidos por meio de buscas eletrônicas realizadas nos CDROMs das edições dos eventos propostos, tendo em vista o fácil acesso aos mesmos. Para a seleção dos artigos analisados, realizou-se uma pesquisa com as seguintes palavras-chave: Hospital, hospitalar, hospitalares, hospitais, saúde, custos, custeio e serviços. Quando não havia possibilidade de consulta por palavra-chave, os artigos publicados foram analisados individualmente, por meio da leitura criteriosa do título e dos resumos, para verificar se o trabalho poderia ou não integrar a amostra.

Na Tabela 1, o universo da pesquisa no período escolhido, bem como a amostra dos artigos selecionados para a análise proposta.

Tabela 1 - Trabalhos Publicados por Evento

\begin{tabular}{c|c|c|c|c}
\hline Ano & Evento & População & Artigos Selecionados & \multirow{2}{*}{ Percentual* $^{*}$} \\
\hline \multirow{4}{*}{2007} & USP* & 155 & 1 & \multirow{2}{*}{$20,87 \%$} \\
\cline { 2 - 4 } & EnANPAD & 975 & 1 & \\
\cline { 2 - 4 } & CBC & 238 & 10 & \\
\cline { 2 - 4 } & ANPCONT & 47 & 1 & $21,59 \%$ \\
\hline \multirow{2}{*}{2008} & USP* & 122 & 0 & \\
\cline { 2 - 4 } & &
\end{tabular}




\begin{tabular}{|c|c|c|c|c|}
\hline & EnANPAD & 1019 & 0 & \\
\hline & $\mathrm{CBC}$ & 276 & 6 & \\
\hline & ANPCONT & 47 & 0 & \\
\hline \multirow{4}{*}{2009} & USP* & 85 & 0 & \multirow{4}{*}{$19,10 \%$} \\
\hline & EnANPAD & 890 & 0 & \\
\hline & $\mathrm{CBC}$ & 253 & 2 & \\
\hline & ANPCONT & 67 & 0 & \\
\hline \multirow{4}{*}{2010} & USP* & 123 & 3 & \multirow{4}{*}{$19,21 \%$} \\
\hline & EnANPAD & 822 & 0 & \\
\hline & $\mathrm{CBC}$ & 277 & 10 & \\
\hline & ANPCONT & 81 & 0 & \\
\hline \multirow{4}{*}{2011} & USP* & 145 & 1 & \multirow{4}{*}{$19,23 \%$} \\
\hline & EnANPAD & 869 & 2 & \\
\hline & $\mathrm{CBC}$ & 195 & 4 & \\
\hline & ANPCONT & 95 & 0 & \\
\hline & Total & 6781 & 41 & $100,00 \%$ \\
\hline
\end{tabular}

*Percentual relativo à população total

*A população do congresso USP corresponde à somatória do Congresso de Controladoria e Contabilidade e de Iniciação Científica em Contabilidade

Fonte: Pesquisa realizada pelas autoras (2012)

A população do estudo corresponde a 6.781 artigos publicados, sendo 4.575 artigos pertencentes ao EnANPAD, 1.239 ao CBC, 630 ao USP e 337 ao Anpcont. Os artigos publicados no Congresso USP de Controladoria e Contabilidade foram somados aos artigos do Congresso USP de Iniciação Científica, visto que os dois eventos são realizados, anualmente, em conjunto, no mesmo período.

Após o levantamento dos artigos, foram selecionados 41 trabalhos científicos com o tema custos na área hospitalar, os quais constituem a amostra desta pesquisa. Na Tabela 2, apresentam-se as distribuições dos artigos selecionados por ano, em cada evento.

Tabela 2 - Publicações Selecionadas por Ano e Fonte

\begin{tabular}{c|c|c|c|c|c|c}
\hline $\begin{array}{c}\text { Ano de } \\
\text { Publicação }\end{array}$ & USP & EnANPAD & CBC & Anpcont & Total & Percentual \\
\hline 2007 & 1 & 1 & 10 & 1 & 13 & $31,71 \%$ \\
\hline 2008 & 0 & 0 & 6 & 0 & 6 & $14,63 \%$ \\
\hline 2009 & 0 & 0 & 2 & 0 & 2 & $4,88 \%$ \\
\hline 2010 & 3 & 0 & 10 & 0 & 13 & $31,71 \%$ \\
\hline 2011 & 1 & 2 & 4 & 0 & 7 & $17,07 \%$ \\
\hline Total & 5 & 3 & 32 & 1 & 41 & $100,00 \%$ \\
\hline Percentual & $12,19 \%$ & $7,32 \%$ & $78,05 \%$ & $2,44 \%$ & $100,00 \%$ & \\
\hline
\end{tabular}

Fonte: Pesquisa realizada pelas autoras (2012)

Verifica-se, na análise dos resultados (Tabela 2), que o $\mathrm{CBC}$ tem os maiores percentuais de publicações na área de custos em hospitais, nos cinco anos analisados. Observa-se que a quantidade das publicações no CBC varia bastante, pois, no ano de 2007, foram publicados 10 artigos, havendo uma queda significativa da produção científica referente ao tema, nos dois anos subsequentes. Comparando-se com o ano de 2007, a produção científica no tema decresceu $40 \%$ em 2008 e, no ano de 2009, 80\%. Já no ano de 2010, crescem as publicações, voltando a cair no ano de 2011. 
Os congressos USP, EnANPAD e AnpCont não tiveram publicações referentes ao tema nos anos 2008 e 2009, sendo a publicação bem retraída nos anos 2010 e 2011. Percebese que a produção científica com abordagem na área de custos hospitalares é pequena em nosso país. O Gráfico 1 apresenta a produção científica em custos no setor hospitalar no período em estudo.

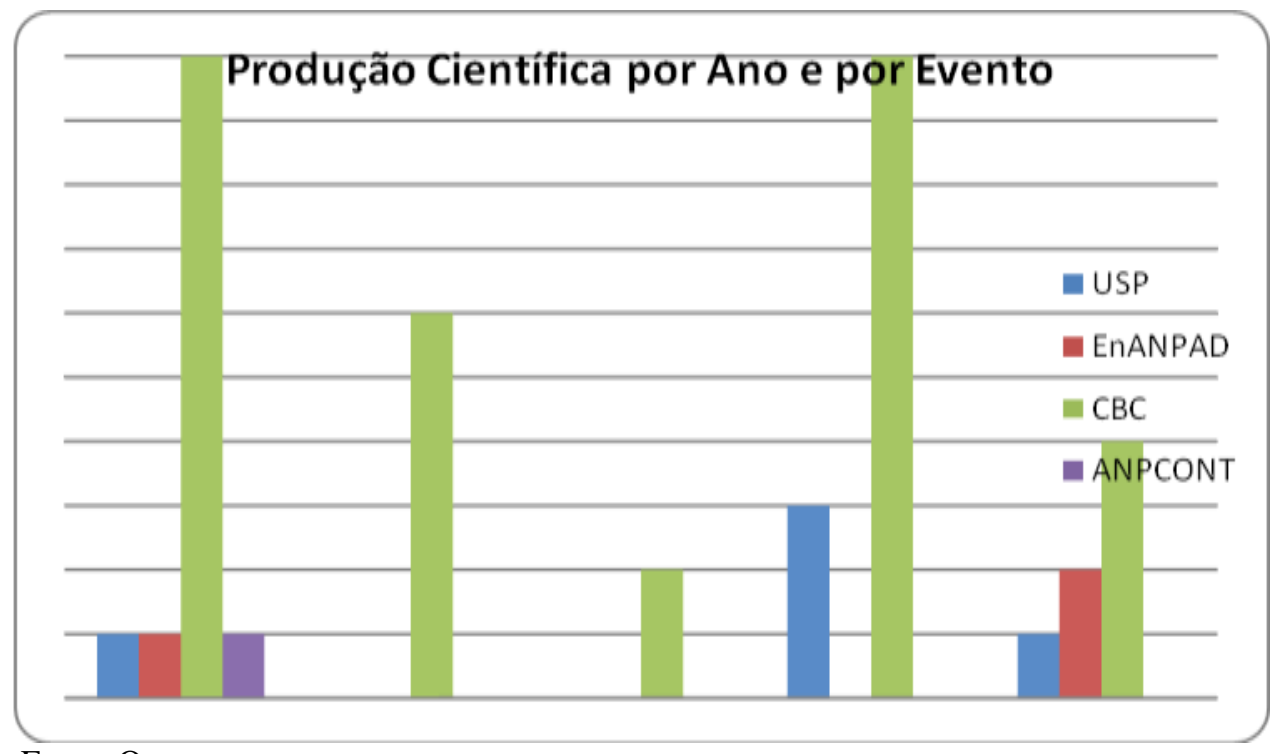

Fonte: Os autores

\section{Gráfico 1: Produção Científica por Evento}

Conforme mencionado anteriormente, a maior publicação na área de custos em hospitais pertence ao $\mathrm{CBC}$. Os resultados apresentam a volatilidade das publicações no $\mathrm{CBC}$ e a escassez de produção referente ao tema nos outros eventos analisados.

\section{ANÁLISE QUALITATIVA DOS DADOS}

No Quadro 1, apresentam-se os 41 artigos selecionados, tendo 1 artigo sido excluído da amostra pelo mesmo estar publicado em dois eventos. Assim, a amostra analisada corresponde a 40 trabalhos.

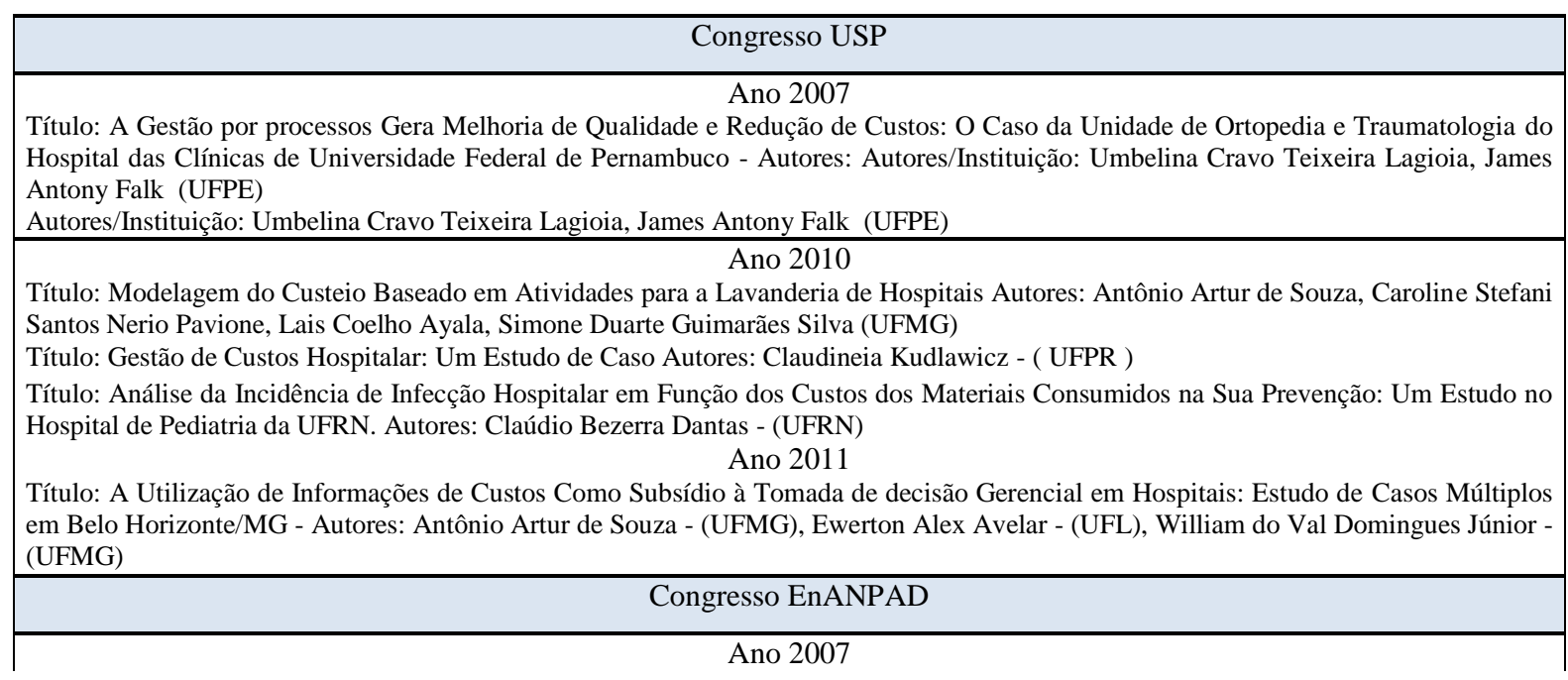


Título: Modelagem do Custeio Baseado em Atividades para Farmácias Hospitalares - Autores: Antônio Artur de Souza (UFMG) Natália Cardoso de Souza (UFMG), Simone Letícia Raimundini (UFRGS), Fabrícia de Farias da Silva, Eduardo Teixeira Valverde Ano 2011

Título: Gestão de custos Interorganizacionais: Evidências Empíricas em Hospitais Privados no Brasil - Autores: Reinaldo Rodrigues Camacho e Romildo de Oliveira Moraes (UEM)

Título: Custeamento ABC Numa Organização Hospitalar Privada: Um Estudo Comparativo do Custo de Cirurgias Eletivas com os valores Reembolsados pelos Planos de Saúde - Autores: Domingos Fernandes Campos e Isabel Cristina Pansiera Marques

\section{Ano 2007}

Título: Cálculo de Custos em Hospitais: Um Estudo de Caso Aplicado na Maternidade de um Hospital Universitário - Autores/Instituição: Marcia Zanievicz da Silva - (UERJ), Altair Borget - (UFSC), Charles Albino Schultz - (UFSC)

Título: Uma Aplicação do Custeio Baseado em Atividades na Central de Materiais Esterilizados de Hospitais - Autores: Antônio Artur de Souza (UFMG) Natália Cardoso de Souza (UFMG), Simone Letícia Raimundini (UFRGS), Miriam Aparecida Micarelli Struett (FCV) Título: Gestão por Processos Gera Melhoria de Qualidade e Redução de Custos: O Caso da Unidade de Ortopedia e Traumatologia do Hospital das Clínicas da Universidade Federal do Pernambuco - Autores: Umbelina Cravo Teixeira Lagioia (UFPE); José Francisco Ribeiro Filho (UFPE); Jorge Expedito de Gusmão Lopes (UFPE); Marcleide Maria Macedo Pederneiras (UFCG

Título: Análise da Influência Tecnológica sobre a Formação de Curso de serviço de Ressonância Magnética em Entidades Hospitalares: Um Estudo Comparativo - Autores: Josicarla Soares Santiago (UFRN); César Augusto Tibúrcio Silva - (UnB; UFPE; UFPR; UFRN) Título: A Utilização das Informações de Custos por Instituições Hospitalares: Um Estudo em Hospitais da Cidade de Natal-RN - Autores: Josicarla Soares Santiago (UFRN); Fátima Gorete Soares de Souza Lopes - (Faculdade União Americana); Diogo Henrique Silva de Lima; Yara Magaly Albano Soares - (UFRN)

TítuloAnálise dos custos da Cirurgia Bariátrica em Comparação aos recursos financeiros Repassados pelo Sistema Único de Saúde (SUS): Estudo de Caso em um Hospital Universitário na Cidade de Maceió/AL - Autores: Carla Monique de Lima; Antônio Carlos S. Costa; Altair de Almeida Campos; Guilherme Costa Farias - (UFAL)

Título: Análise do Comportamento dos Custos Hospitalares Indiretos: Uma Investigação Empírica do Custo Hospitalar de Energia Elétrica no Setor de Radioterapia da Liga Norte-Rio-Grandense Contra o Câncer - Autores/Instituição: Iana Silva Gomes (UFRN); Diogo Henrique Silva de lima (UnB; UFPB; UFPE; UFRN); Adriana Isabel Backes Steppan (UFRN)

Título: Aplicabilidade do Custeio em Atividades e Análise de custos em Organizações Hospitalares - Autores: Edvalda Araújo Leal; Roberto Fernandes dos santos; Neusa Maria Bastos F. Santos - (PUC-SP)

Título: Gestão de Custos Hospitalares por meio do Custeio por Absorção: O Caso da Maternidade Carmela Dutra - Autores Cláudia Hernandes Crispim (UDESC); Éder da Silveira de Almeida (UFSC); Fabiano Maury Raupp (UDESC)

Título: Estratégia, Custos e Hospitais: Um Diagnóstico na Cidade de Salvador, Bahia (2006) - Autores: Alexandre David da Silva Oliveira; Adriano Leal Bruni; Roberto Brasileiro Paixão - (UNIFACS); Cesar Valentim Carvalho Júnior - (UNIFACS e UFBA)

Ano 2008

Título: Discriminação de preços aos trancos e Barrancos e sem uso de Métodos de Custeio Adequados: Evidências de dois Hospitais do Município do Rio de Janeiro - Autores: Elton Azevedo Maia; Ricardo Lopes Cardoso (FGV/EBAPE)

Título: Descrição do custeio Baseado em Atividades para o Serviço de Nutrição e Dietética Hospitalar - Autores: Antônio Artur de Souza; Mariana Guerra; Thaís Las-Casas Macedo Amorim; Éllen Santos de Moraes (UFMG); Leticia Maria de Lacerda Moreira Pena (FNH) Título: Sistema de Custos para o Centro de Diagnóstico por Imagens do Hospital Universitário Onofre Lopes - Autores: Raquel Esperanza Patino-escarcina; Dennis Barrios Aranibar; Benjamim Rene Callejas Bedregal (UFRN); Ivanosca Andrade da Silva (HUOL - UFRN) Título: Análise e Estatística de Custos em Organizações Hospitalares: Um Estudo Comparativo entre Hospital Geral e Maternidade Autores/Instituição: Cláudia Hernandez Crispim (UFSC/IES); Altair Borget; Éder da Silveira de Almeida - (UFSC)

Título: Fatores que Influenciam a Redução de Custos com Enfoque no Tempo de Permanência do paciente em uma Instituição Hospitalar - Autores Geize Ketlin O. da Silva; Rosimere Alves de Bona Porton; Kátia Aurora Dalla Libera Sorato; Daniela Martinello - (UNESC) Título: Perfil dos Artigos de Custos Voltados para área Hospitalar Publicados nos Anais do Congresso USP período de 2001-2007 Autores: Wenner Glaucio Lopes Lucena; Rommel de Santana Freire; Leide Adriana da silva Neri Brito - (UFPB) Ano 2009

Título: O Sistema Estadual de Monitoramento dos Centros Viva Vida e as Bases para a Implantação dos Sistemas de Custos na Atenção Secundária à Saúde em Minas Gerais - Autores Alisson Maciel de Faria Marques; Hellen Fernanda Souza; Laura Monteiro de Castro Moreira - (SESMG)

Título: Proposta de Metodologia para a Implantação do sistema de Custeio Baseado em Atividades para organizações Hospitalares Autores: Antônio Artur de Souza; Mariana Guerra; Ewerton Alex Avelar - (UFMG)

Ano 2010

Título: Implantação do sistema de custos na maior rede hospitalar da América Latina: o caso da Fundação Hospitalar do Estado de Minas Gerais (FHEMIG) - Autores: Márcio Mascarenhas Alemão; Josiano Gomes Chaves; Heloisa Azevedo Drumond; Dayenne Cristina Martins Valente - (FHEMIG)

Título: Comportamento dos Custos em Hospitais Administrados pela Secretaria de Estado da saúde de Santa Catarina - Autores: Cláudia Hernandez Crispim (UFSC/IES); Altair Borget; Éder da Silveira de Almeida - (UFSC)

Título: Critérios de Classificação de Custos quanto ao Comportamento Aplicado em uma Entidade Hospitalar: Uma Análise das divergências entre métodos utilizados e o seu impacto no resultado - Autores: Luiz Claúdio Louzada, Franklim Dantas Oliveira, Flávio Boldrini Mulinari, Eduardo José Zanoteli, Jean Pierre David de Oliveira

Título: Custo do Parto Normal e Cesáreo: Replicação do Custeio ABC - Autores: Erivelto Martins Paixão, Antônio Artur de Souza, Livia Carolina de Matos Lima - (UFMG)

Título: O custeio alvo em serviços hospitalares públicos: estudo no serviço de transplante hepático do Hospital Universitário Walter Cantídio da Universidade Federal do Ceará (HUWC/UFC) - Autores: Marcos Antônio Barbosa de Lima, Maria da Gloria Arrais Peter, Marcus Vínícios Veras Machado, Anelise Florencio de Meneses - (UFC), Ana Maria Fontenelle Catrib (UNIFOR)

Título: Gestão de Custos Hospitalares em Instituição Pública: Um Estudo de Caso - Autores: Tânia Maria Stoffel (UFMT/CUR), José Ribeiro Viana Filho, Izabel Augusta Moreira de Oliveira, Bruno Pereira dos santos

Título: Custos na Prestação de Serviços: Uma Aplicação do Custo por Departamento em uma Organização Hospitalar - Autores:

Claudineia Kudlawicz, Ely Célia Corbari (UFPR) 
Fonte: Pesquisa realizada pelas autoras (2012)

\section{Quadro 1 - Amostra dos Artigos Publicados na área de Custos em Hospitais}

Para a análise qualitativa dos artigos selecionados, organizou-se, para cada trabalho, uma ficha-resumo destinada à identificação, ao registro e ao armazenamento das informações. Buscou-se localizar a origem e tipo de trabalho, as questões que vêm despertando maior interesse dos pesquisadores nacionais na área de custos hospitalar, a orientação predominante em termos de referencial teórico, além de descrever as principais características metodológicas dos trabalhos publicados.

A ficha-resumo foi organizada com os seguintes dados: o título e identificação do artigo; as questões de pesquisa; os objetivos; a metodologia e os resultados encontrados. Os dados foram analisados a partir de dimensões analíticas que reuniram informações de um ou mais campos da ficha-resumo dos artigos.

Os resultados serão apresentados na seguinte ordem: abordagens adotadas nas pesquisas; a origem institucional dos trabalhos publicados; as publicações por autores; e as características e abordagens metodológicas dos artigos.

\subsection{Abordagens e Origem Institucional dos Trabalhos}

No Quadro 2, estão resumidas as diversas abordagens referentes à aplicação dos métodos de custeio, encontradas em 20 artigos analisados:

\begin{tabular}{|c|c|}
\hline Quantidade de Artigos & Método de Custeio Aplicado \\
\hline 11 & Custeio Baseado em Atividades \\
\hline 4 & Custeio por Absorção \\
\hline 2 & Custos por Departamento \\
\hline 1 & Custeio Variável \\
\hline 1 & Custeio Alvo \\
\hline 1 & Método Híbrido \\
\hline
\end{tabular}

Fonte: Pesquisa realizada pelos autores (2012)

Quadro 2 - Métodos de Custeio Aplicados nas Pesquisas

Diversos trabalhos analisados fazem a comparação dos diversos métodos de custeio nas organizações hospitalares. 
Para a análise da instituição de ensino nas publicações, foram utilizados os seguintes critérios: (1) para os artigos cujos autores estão vinculados a diferentes instituições, selecionou-se a instituição que tem o maior numero de autores; (2) no caso dos artigos cujos autores estão vinculados a diferentes instituições, sem a repetição da instituição por autor, selecionou-se o nome do primeiro autor.

No Gráfico 2, apresenta-se o percentual de publicações por instituição de ensino, considerando a origem institucional dos autores.

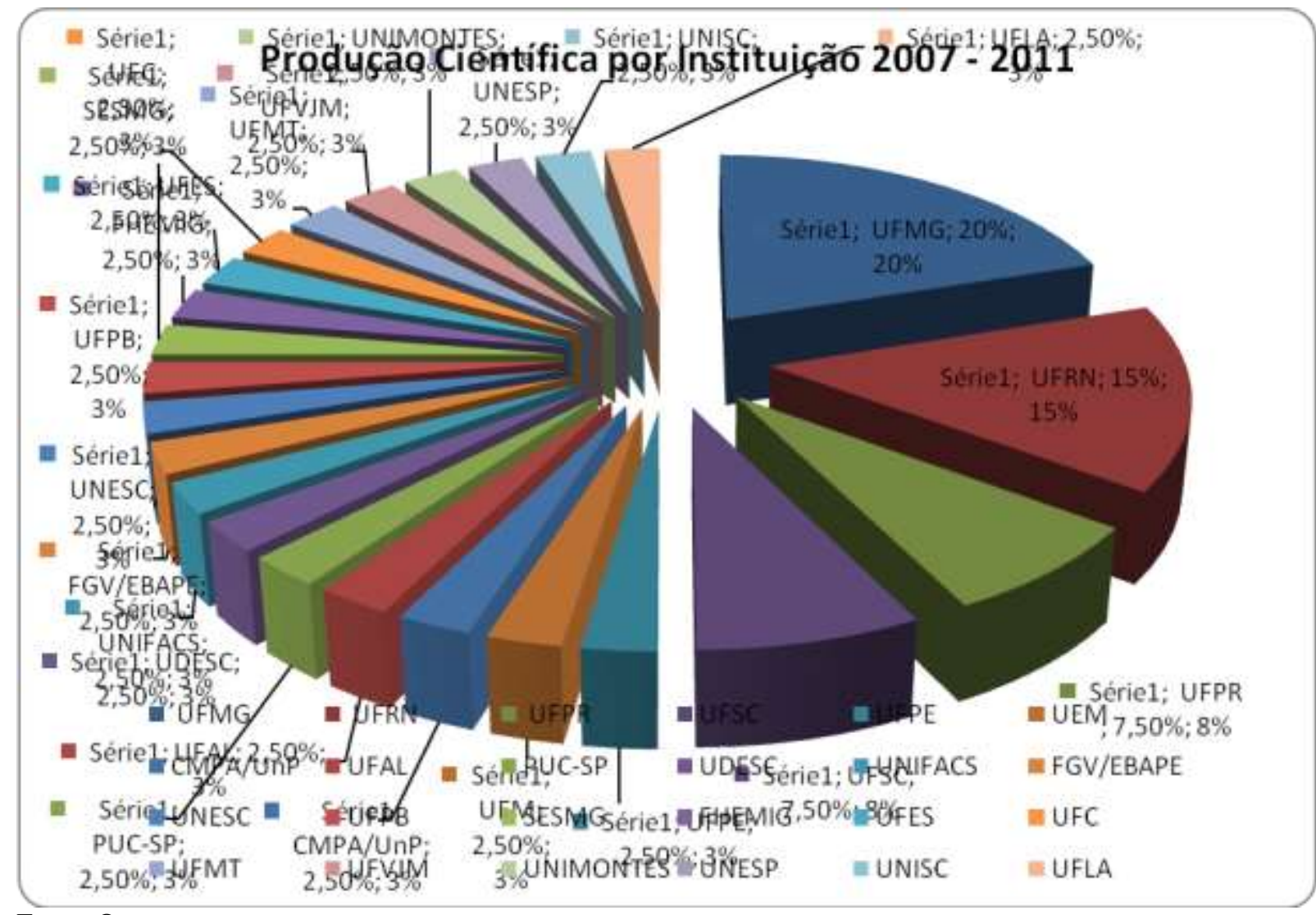

Fonte: Os autores

Gráfico 2: Origem Institucional das Publicações

A instituição com o maior número de artigos publicados em Custos na área Hospitalar, no período analisado, foi a Universidade Federal de Minas Gerais (UFMG), com 8 artigos publicados, destacando-se com $20 \%$ das 40 publicações. Em seguida, com 6 publicações, está a Universidade Federal do Rio Grande do Norte (UFRN); com 15\%, a Universidade Federal do Paraná (UFPR) e a Universidade Federal de Santa Catarina (UFSC) publicaram 3 artigos cada, correspondendo a 7,5\% das publicações. As demais Universidades publicaram 1 artigo, perfazendo $2,5 \%$ do total das produções acadêmicas

A tabela 3 apresenta a quantidade de autores por artigo publicado. Observa-se que a maioria dos autores prefere desenvolver as pesquisas em parceria com 2, 3, 4 e, até mesmo, 5 autores, sendo a autoria, na maior parte das publicações $(37,50 \%)$, composta por um conjunto de 3 autores por artigo. Não há entre os autores uma característica predominante em publicar individualmente seus trabalhos, sendo essa opção de apenas 2 artigos, correspondendo a 5\% do total. 
Tabela 3 - Número de Autores por Publicação

\begin{tabular}{ccc}
\hline Número de Autores & Artigos & Percentual (\%) \\
\hline 1 & 2 & $5,00 \%$ \\
2 & 8 & $20,00 \%$ \\
3 & 15 & $37,50 \%$ \\
4 & 9 & $22,50 \%$ \\
5 & 6 & $15,00 \%$ \\
Total & 40 & $100,00 \%$ \\
\hline
\end{tabular}

Fonte: Pesquisa realizada pelas autoras (2012)

Dos 129 autores evidenciados na Tabela 4, apenas 13 tiveram mais de 1 artigo publicado no tema abordado nesta pesquisa, no período de 2007 a 2011, correspondendo a $10,08 \%$.

Tabela 4 - Número de Publicações por Autor

\begin{tabular}{ccc}
\hline Autores & Número de Autores & Percentual (\%) \\
\hline 1 artigo publicado & 116 & $89,92 \%$ \\
2 artigos publicados & 9 & $6,98 \%$ \\
3 artigos publicados & 3 & $2,32 \%$ \\
4 artigos publicados & 0 & $0,00 \%$ \\
5 artigos publicados & 0 & $0,00 \%$ \\
6 artigos publicados & 0 & $0,00 \%$ \\
7 artigos publicados & 0 & $0,00 \%$ \\
8 artigos publicados & 1 & $0,78 \%$ \\
\hline Total & 129 & $100,00 \%$ \\
\hline
\end{tabular}

Fonte: Pesquisa realizada pelas autoras (2012)

\subsection{ABORDAGENS METODOLÓGICAS DOS ARTIGOS}

Machado-da-Silva; Cunha; Amboni (apud Tonelli et al, 2003, p.109) apresentam a classificação para estudos empíricos e/ou teóricos. Para os autores, os artigos podem ser classificados em: (1) teórico-empíricos: compreendendo estudos que partem de um quadro de referências teóricas e, pela coleta de dados, buscam confirmá-los ou refutá-los, no todo ou em parte; e (2) teóricos: envolvendo trabalhos que se limitam a conceitos, proposições, identificação de variáveis, construção ou reconstrução de modelos, sem envolver teste empírico.

Nesse contexto, os trabalhos analisados foram separados por abordagens metodológicas considerando a classificação apresentada. A tabela 5 apresenta as metodologias utilizadas nos artigos pesquisados, no período de 2007 a 2011, analisando-se cada artigo com o objetivo de verificar as classificações metodológicas quanto aos objetivos, abordagens, procedimentos e métodos utilizados para coleta dos dados. 
Revista de Administração Hospitalar, v.11, n.1, pp. 42-57, janeiro/março, 2014/ Maria Eliana dos Santos, Edvalda Araújo Leal e Davy Antônio da Silva

Tabela 5 - Metodologia Utilizada nos Artigos

\begin{tabular}{|c|c|c|}
\hline Metodologias & \multirow[t]{2}{*}{ Artigos } & \multirow[t]{2}{*}{ Percentua } \\
\hline & & \\
\hline Empírico & 38 & $95,00 \%$ \\
\hline Teórico-empírico & 1 & $2,50 \%$ \\
\hline Bibliométrico & 1 & $2,50 \%$ \\
\hline Total & 40 & $100 \%$ \\
\hline \multicolumn{3}{|c|}{ Objetivo } \\
\hline Descritiva & 18 & $45,00 \%$ \\
\hline Não divulgou & 10 & $25,00 \%$ \\
\hline Exploratória & 8 & $20,00 \%$ \\
\hline Exploratória/Descritiva & 3 & $7,50 \%$ \\
\hline Bibliográfica/Documental & 1 & $2,50 \%$ \\
\hline Total & 40 & $100,00 \%$ \\
\hline \multicolumn{3}{|c|}{ Abordagem } \\
\hline Qualitativa & 22 & $55,00 \%$ \\
\hline Qualitativa/Quantitativa & 8 & $20,00 \%$ \\
\hline Quantitativa & 7 & $17,50 \%$ \\
\hline Não divulgou & 3 & $7,50 \%$ \\
\hline Total & 40 & $100,00 \%$ \\
\hline \multicolumn{3}{|c|}{ Procedimentos } \\
\hline Estudo de Caso & 25 & $62,50 \%$ \\
\hline Levantamento/Survey & 6 & $15,00 \%$ \\
\hline Estudo de Caso múltiplo & 4 & $10,00 \%$ \\
\hline Estudo de Caso/Quase experimento & 1 & $2,50 \%$ \\
\hline Expost-facto/Estudo de Caso & 1 & $2,50 \%$ \\
\hline Estudo de Caso/Bibliográficos/Documental & 1 & $2,50 \%$ \\
\hline Estudo de Caso único & 1 & $2,50 \%$ \\
\hline Não divulgou & 1 & $2,50 \%$ \\
\hline Total & 40 & $100 \%$ \\
\hline \multicolumn{3}{|c|}{ Coleta de Dados } \\
\hline Pesquisa Documental & 16 & $40,00 \%$ \\
\hline Entrevista & 10 & $25,00 \%$ \\
\hline Entrevista, Pesquisa documental/Observação & 3 & $7,50 \%$ \\
\hline Entrevista/Observação não participante & 2 & $5,00 \%$ \\
\hline Não divulgou & 2 & $5,00 \%$ \\
\hline Questionário & 2 & $5,00 \%$ \\
\hline Entrevista/ Pesquisa documental & 2 & $5,00 \%$ \\
\hline Entrevista /Grupo focal/ Observação Participante & 1 & $2,50 \%$ \\
\hline Entrevista /Questionário & 1 & $2,50 \%$ \\
\hline Análise Descritiva e Documental & 1 & $2,50 \%$ \\
\hline
\end{tabular}




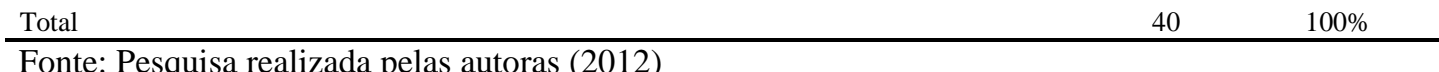

Dos 40 artigos pesquisados, $95 \%$ são classificados como empíricos, indicando que as publicações científicas na área de custos em organizações hospitalares optam por aplicação prática. $\mathrm{Na}$ análise da categorização dos objetivos das pesquisas, observou-se um percentual significativo de pesquisas descritivas (45\%), seguidas por pesquisas exploratórias (20\%). Verificou-se, ainda, que $25 \%$ dos trabalhos não divulgaram a classificação metodológica quanto aos objetivos pesquisados.

Quanto à abordagem adotada nos trabalhos, o maior percentual foi para a pesquisa qualitativa (55\%), seguida por quali-quanti (20\%). Acredita-se que a escolha de tais abordagens para análise das pesquisas deve-se ao percentual significativo da utilização do estudo de caso como procedimento de pesquisa (62,5\%), tanto estudo de caso único como de multi-casos.

Para a coleta de dados, verificou-se a predominância da utilização da pesquisa documental (40\%) e aplicação de entrevistas (25\%). Percebeu-se que muitos trabalhos utilizam mais de um procedimento para a coleta de dados, o que é indicado por autores de metodologia. Beuren (2008) recomenda, para estudos com abordagem qualitativa, o uso de múltiplas fontes de evidências, aplicando-se métodos de coleta de dados combinados como entrevistas, questionários e observação.

\section{CONSIDERAÇÕES FINAIS}

Este estudo teve como objetivo identificar o perfil das pesquisas relacionadas ao tema de custos com ênfase na área hospitalar publicadas nos principais eventos científicos na área contábil, no período compreendido entre o ano de 2007 a 2011. O universo da pesquisa corresponde a 6.781 artigos publicados no período, tendo sido selecionados 40 trabalhos com o tema em estudo, representando menos de $1 \%$ das publicações.

Verificou-se que o tema custos no contexto hospitalar é ainda pouco explorado na academia. Dos eventos pesquisados, o Congresso Brasileiro de Custos foi o que apresentou o maior número de publicações sobre o tema. Já nos outros congressos (USP, EnANAPD e AnpCont), a produção no tema ainda é limitada.

Na classificação das publicações por origem institucional dos autores, verificou-se que o maior volume refere-se às publicações de autores vinculados à Universidade Federal de Minas Gerais, com destaque, também, para a Universidade Federal do Rio Grande do Norte. Evidenciou-se, com esta pesquisa, que os autores das publicações investigadas na área de custos em hospitais escolhem desenvolver as pesquisas em conjunto com outros autores, sendo $95 \%$ das publicações realizadas em parceria.

Foi realizada a análise qualitativa detalhada de todos os trabalhos selecionados, com o objetivo de verificar as classificações metodológicas adotadas. A análise considerou os objetivos, abordagens, procedimentos e os métodos utilizados para coleta dos dados. Verificou-se que $95 \%$ dos artigos utilizam aplicação empírica, reforçando o interesse dos autores em verificar a aplicabilidade da gestão de custos nas organizações hospitalares.

Quanto à identificação da tipologia das pesquisas, o percentual mais significativo é do tipo descritivo, seguido de estudos exploratórios. Verificou-se que $25 \%$ dos trabalhos não 
identificaram a classificação metodológica quanto ao tipo de pesquisa. A abordagem predominante nos artigos analisados foi a qualitativa, seguida de quali-quanti, o que pode ser justificado pela utilização predominante do procedimento de estudo de caso $(62,5 \%)$. Na análise da coleta de dados, observou-se que o maior número de trabalhos utilizou a entrevista e pesquisa documental.

Conclui-se, com esta pesquisa, que as pesquisas sobre os temas relacionados à gestão de custos, especificamente, em organizações hospitalares, são, ainda, em quantidade bastante limitada, o que chama a atenção para a necessidade de novas pesquisas nessa área. Indica-se, para futuras pesquisas, a análise das publicações em periódicos nacionais na área contábil, para avaliar o perfil de divulgação dos trabalhos acadêmicos que abordam esse tema.

\section{REFERÊNCIAS:}

ABBAS, Kátia. Gestão de custos em organizações hospitalares. Florianópolis, 2001. Dissertação (Mestrado em Engenharia de Produção) - Programa de Pós Graduação em Engenharia de Produção, Universidade de Santa Catarina.

ALMEIDA, Teófilo R. R. Perspectivas de sobrevivência do hospital. Revista Paulista de Hospitais, São Paulo, n.5/6, p.104-113, maio/jun. 1983.

AZEVEDO, C.S. Gerência hospitalar: a visão dos diretores de hospitais públicos do município do Rio de Janeiro. Rio de Janeiro, 1993. Dissertação de mestrado. Instituto de Medicina Social, Universidade Federal do Rio Janeiro

CALlADO, Aldo Leonardo Cunha; ALMEIDA, Moisés Araújo. Perfil dos artigos sobre custos no agronegócio publicados nos anais do Congresso Brasileiro de Custos. Custos e agronegócio on-line. v.1, n.1, p. 1-20. jan-jun 2005. Disponível em <http://www.custoseagronegocioonline.com.br/numeroinaugural.html> 24 jun 2005.

CAMPOS, D. F; MARQUES, I. C; Custeamento ABC numa Organização Hospitalar Privada: Um Estudo Comparativo do Custo de Cirurgias Eletivas com os Valores Reembolsados pelos Planos de Saúde. ENANPAD, 2011

CARDOSO, Ricardo Lopes; PEREIRA, Carlos Alberto; GUERREIRO, Reinaldo. A Produção Acadêmica em Custos no Âmbito do ENANPAD: uma Análise de 1998 a 2003. In: ENCONTRO ANUAL DA ASSOCIAÇÃO NACIONAL DOS PROGRAMAS DE PÓSGRADUAÇÃO EM ADMINISTRAÇÃ̃O, 28, Anais. Curitiba: ENANPAD, 2004

CHING, H. Y. Manual de custos de instituições de saúde: sistemas tradicionais de custos e sistema de custeio baseado em atividades (ABC). 2 ed. São Paulo: Atlas, 2010.

FALK, J. A. Gestão de custos para hospitais: conceitos, metodologias e aplicações. São Paulo: Altas, 2001.

GIL, A.C. Métodos e técnicas de pesquisa social. São Paulo: Atlas, 2011

HJORLAND, B; Core Concepts in Library and Information Science (LIS); Disponível em: <http://www.db.dk/bh/core\%20concepts\%20in\%20lis/home.htm>. Acesso em: Julho 2007

LAKATOS, Eva Maria; MARCONI, Marina de Andrade. Metodologia do trabalho cientifico. 4 ed. São Paulo: Atlas, 1995. 
LUCENA, W. G. L.; BRITO, L. A. da S. N.; FREIRE, R. de Santana . Perfil dos artigos sobre custos voltados para área hospitalar publicados nos anais do congresso USP período de 2001-2007. In: XV Congresso Brasileiro de Custos, 2008, Curitiba. XV Congresso Brasileiro de Custos, 2008.

MACHADO, Lisiane; SILVA, Lisiane Vasconcellos da. A Pesquisa Acadêmica no Contexto Internacional - Uma Análise Exploratória dos Trabalhos de Conclusão de Curso, desenvolvidos na Graduação em Administração com Habilitação em Comércio Exterior, em uma Universidade do Sul do País. In: ENCONTRO NACIONAL DA ASSOCIAÇÃO NACIONAL DOS PROGRAMAS DE PÓS-GRADUAÇÃO EM ADMINISTRAÇÃO, 31, 2007, Rio de Janeiro, Anais Eletrônicos do Encontro Anual da ANPAD. Rio de Janeiro: ANPAD, 2007. p. 01-16.

MACHADO DA SILVA, Clóvis L.; CUNHA, Vera; AMBONI, Nério. Organizações: o estado da arte da produção acadêmica. In. Anais do Encontro Anual da Associação Nacional dos Programas de Pós-graduação em Administração, 14, Florianópolis: ANPAD, 1990. p. 1128.

MARCONI, M. A., LAKATOS, E. M. Fundamentos de metodologia científica. 3. ed. São Paulo: Atlas, 1991.

MARTINS, Domingos. Custos e Orçamentos Hospitalares. São Paulo: Atlas, 2000.

MARTINS, E. Contabilidade de Custos. 9 ed. São Paulo: Atlas, 2003.

MATOS, A. J. Gestão de custos hospitalares: técnicas, análise e tomada de decisão. São Paulo: STS, 2002

PRITCHARD, A. Statistical bibliography or bibliometrics? Journal of Documentation, v. 25, n. 4, p. 348-349, Dec. 1969

RICHARDSON, Roberto Jarry. Pesquisa Social: métodos e técnicas. 3. ed. São Paulo: Atlas, 1999

ROCHA, D. T.; REIS, J. A. F. dos; SOUZA, A. ; CRUZ, J. A. W. ; TRACZ, L.. Gestão de custos: um estudo bibliométrico e sociométrico da produção científica do 1997-2008. In: XVII Congresso Brasileiro de Custos, 2010, Belo Horizonte - MG. XVII Congresso Brasileiro de Custos, 2010

SCHULTZ, Charles Albino; ZANIEVICZ, Márcia; BORGERT, Altair; LAFFIN, Marcos. Produção científica na área de custos no setor de serviços no período de 2000 a 2004. $6^{\circ}$ Congresso de Controladoria e Contabilidade, 499, Anais. São Paulo, 2006

SILVA, M. Z.; BORGET, A.; SCHULTZ, C. A. Sistematização de um método de custeio híbrido para o custeamento de procedimentos médicos: uma aplicação conjunta das metodologias $A B C$ e UEP. Revista de Ciências da Administração, v. 11, n. 23, p. 217-244, 2009

WORMELL, Irene. Informetria: explorando bases de dados como instrumentos de análise. Ciência da Informação, Brasília, v. 27, n. 2, p. 210-216, maio/ago. 1998. Disponível em: <http://www.ibict.br/cionline/>. Acesso em: 15 ago. 2007. 